\title{
Cytokeratin 18 can help predict liver fibrosis in HCV infected patients with type 2 diabetes mellitus
}

Fang $\mathrm{Li}^{1 \dagger}$, Feifei Lei ${ }^{1 \dagger}$, Chengcai Wen ${ }^{2}$, Qiu Ge ${ }^{3^{*}}$ and Liyao Zhu ${ }^{4^{*}}$

\begin{abstract}
Background: To investigate the predictive values of cytokeratin 18 for liver fibrosis in hepatitis $C$ virus $(\mathrm{HCV})$ infected patients with type 2 diabetes mellitus (T2DM).

Methods: 252 HCV-infected patients with T2DM between January 2012 and August 2017 were retrospectively reviewed. Pearson/spearman correlation analysis was used to detect the correlation in the entire cohort. Multivariate linear regression was used to identify independent predictors and logistic regression was for establishing models. Combination models that incorporated CK18 and other methods (i.e. transient elastography, aspartate transaminaseto-platelet ratio index (APRI) and fibrosis-4 index (FIB-4)] were developed in a training cohort of 132 patients. Performance of models was evaluated through discrimination ability and clinical benefits. An internal validation was conducted in 120 consecutive patients.
\end{abstract}

Results: CK18 was found significantly associated with fibrosis scores $(r=0.452, P<.001)$. CK18 and albumin were confirmed as independent predictors for fibrosis. For predicting significant fibrosis in the validation cohort, the observed AUC values of APRI + CK18 (AUC 0.83) and FIB-4 + CK18 (AUC 0.84) were higher than those of APRI (AUC 0.61) and FIB-4 (AUC 0.65). For predicting advanced fibrosis and cirrhosis, the AUC values of FIB-4 + CK18 (AUC 0.74 and 0.77 , respectively) were significantly higher than those of FIB-4 (AUC 0.61 of both). Decision curve analysis confirmed the more clinical benefits can be provided by being combined with CK18.

Conclusions: CK18 is an independent predictor of liver fibrosis for HCV-infected patients with T2DM. Noninvasive methods incorporate CK18 and other biomarker indices can have better performance for diagnosing fibrosis and help clinical decision-making.

Keywords: Hepatitis C virus (HCV), Liver fibrosis, Type 2 diabetes mellitus (T2DM), Cytokeratin 18 (CK18), Predictive values

*Correspondence: gq319329@163.com; zhu_liyao@126.com

${ }^{\dagger}$ Fang Li and Feifei Lei contributed equally as first author

${ }^{3}$ Outpatient Department, The Second People's Hospital of Huai'an, The Affiliated Huai'an Hospital of Xuzhou Medical University, Huai'an, China

${ }^{4}$ Department of Hepatology, The Fourth People's Hospital of Huai'an, Jiangsu, China

Full list of author information is available at the end of the article

\begin{abstract}
Introduction
As estimated by world health organization (WHO) in 2015, 71 million patients were infected with hepatitis $C$ virus (HCV) and 399,000 died because of cirrhosis or hepatocellular carcinoma cause by HCV in the worldwide [1]. About 422 million people worldwide have diabetes mellitus, which have become one of the leading causes of death $[2,3]$. Both $\mathrm{HCV}$ and diabetes mellitus contribute to the global burden of disease.
\end{abstract}

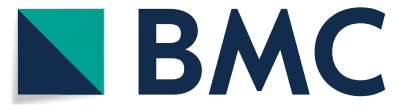

(c) The Author(s) 2021. Open Access This article is licensed under a Creative Commons Attribution 4.0 International License, which permits use, sharing, adaptation, distribution and reproduction in any medium or format, as long as you give appropriate credit to the original author(s) and the source, provide a link to the Creative Commons licence, and indicate if changes were made. The images or other third party material in this article are included in the article's Creative Commons licence, unless indicated otherwise in a credit line to the material. If material is not included in the article's Creative Commons licence and your intended use is not permitted by statutory regulation or exceeds the permitted use, you will need to obtain permission directly from the copyright holder. To view a copy of this licence, visit http://creativecommons.org/licenses/by/4.0/. The Creative Commons Public Domain Dedication waiver (http://creativeco mmons.org/publicdomain/zero/1.0/) applies to the data made available in this article, unless otherwise stated in a credit line to the data. 
Chronic hepatitis $\mathrm{C}(\mathrm{CHC})$ has been found associated with a four-fold increased risk of insulin resistance and type 2 diabetes mellitus (T2DM) [4]. Meanwhile, hyperglycemia and insulin which can stimulate hepatic stellate cells mitogenesis and collagen synthesis, are key factors in the progression of fibrosis [5, 6]. A previous study [7] conducted by Wieckowska et al. showed that cytokeratin 18 (CK18) is at high levels and positively correlated with liver fibrosis stage in patients with non-alcoholic fatty liver disease (NAFLD). Moreover, CK18 has been proposed for direct measures of inflammation in NAFLD patients $[8,9]$.

However, only a few studies investigated the correlations about CK18 in patients infected with $\mathrm{HCV}[10$, 11], and limited data confirmed the strong relationship between serum CK18 and HCV-related fibrosis. Sanyal et al. [10] also found CK18 associated with insulins in $\mathrm{CHC}$ complicated with T2DM. Additionally, there have been many developed noninvasive serum biomarkers for predicting liver fibrosis in $\mathrm{CHC}$ patients without any other metabolic diseases [12], but no marker was specially developed for CHC-T2DM patients. The aim of this study was to investigate the association between the level of CK18 and liver fibrosis and evaluate the predictive value of CK18 in CHC patients with T2DM.

\section{Materials and methods}

This retrospective study was approved by the institutional review board of the Fourth People's Hospital of Huai'an. The requirement for written informed consent was waived by the institutional review board of the Fourth People's Hospital of Huai'an due to its retrospective nature. All methods were performed in accordance with the 1975 Helsinki declaration and its later amendments.

\section{Patients}

The flow chart is shown in Fig. 1. 397 patients diagnosed with $\mathrm{CHC}$ and T2DM, who were admitted to the Fourth People's Hospital of Huai'an between January 2012 and August 2017 were retrospectively reviewed. The inclusion criteria were shown as follows: (a) diagnosed with $\mathrm{CHC}$ (positive anti-HCV antibodies for over 6 months and HCV RNA $>1.0 \times 10^{3} \mathrm{IU} / \mathrm{ml}$ ); (b) clinically determined diabetes; (c) treat-naïve before hospitalization in this hospital. The exclusion criteria were: (a) focal hepatic lesion (i.e. tumor, hepatic tuberculosis and any other); (b) coinfected with other virus such as hepatitis B virus (HBV), hepatitis D virus (HDV) and human immunodeficiency virus (HIV); (c) significant alcohol intake (>20 g/ day); (d) severe hepatic failure (jaundice and ascites or transaminases level more than 10 times the upper limit of normal); (e) inadequate clinical data. Patients were allocated to the training and validation cohort according to the time of hospitalization (Training: January 2012-September 2015; Validation: October 2015-August 2017).

Clinical data included age, sex, body mass index (BMI), plate count (PLT), prothrombin time (PT), international normalized ratio (INR), fasting blood glucose (FBG), fasting insulin (FINS), alanine aminotransferase (ALT), aspartate aminotransferase (AST), alkaline phosphatase

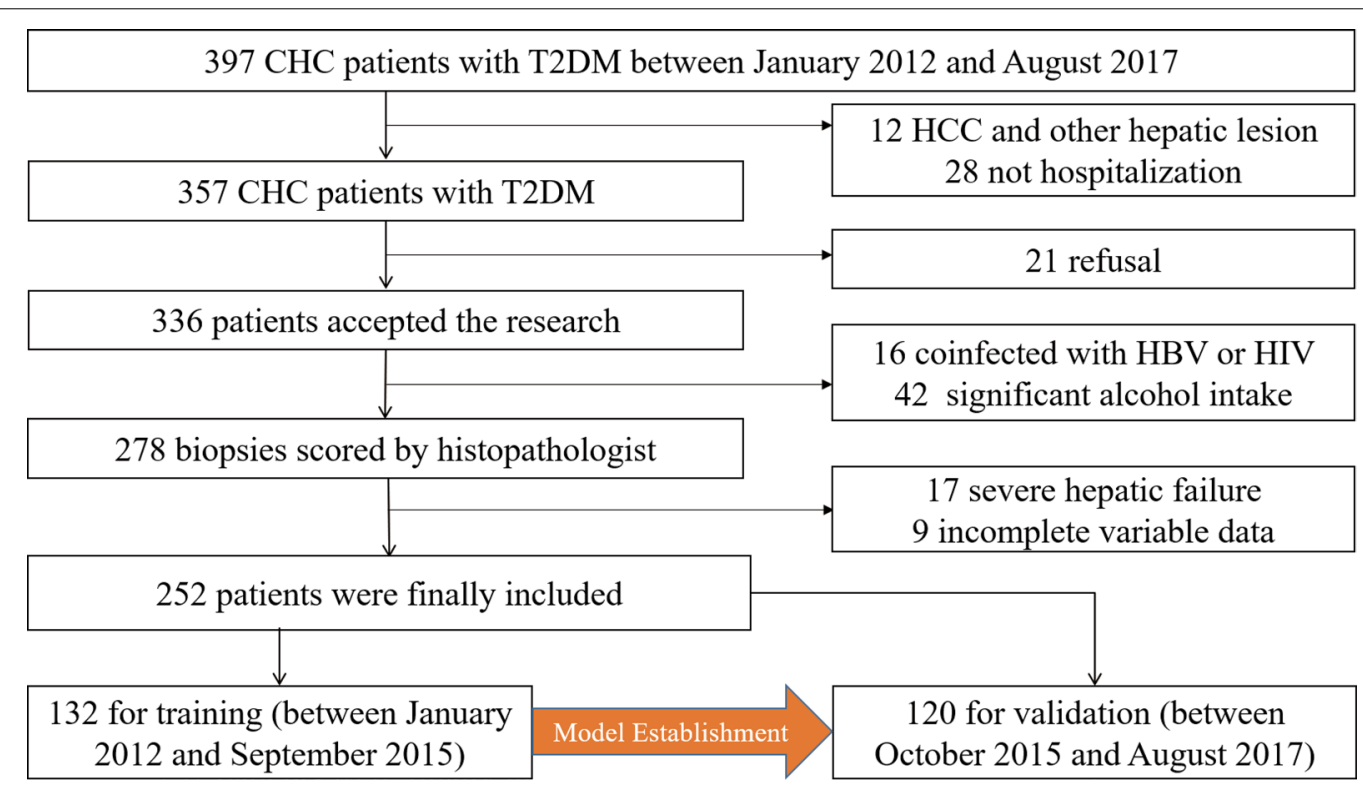

Fig. 1 Patient selection flow chart. CHC, chronic hepatitis C; HBV, hepatitis B virus; HCC, hepatocellular carcinoma; HIV, human immunodeficiency virus 
(ALP), glutamyl transpeptidase (GGT), lactate dehydrogenase (LDH), total cholesterol (TC), triacylglycerol (TG), high density lipoprotein cholesterol (HDL-C) and low density lipoprotein cholesterol (LDL-C) at the first diagnosis of CHB and T2DM. Homeostatic model assessment for insulin resistance (HOMA-IR) score was calculated using the formula: HOMA-IR=FINS $(\mathrm{mIU} / \mathrm{L}) \times \mathrm{FBG}(\mathrm{mmol} / \mathrm{L}) / 22.5$.

\section{Liver biopsy}

Liver biopsy (LB) was performed by experienced ultrasonologists and liver samples were formalin-fixed and paraffin-embedded for analysis. Two senior pathologists, who were blinded to the clinical information, determined final histological results in consensus. Liver fibrosis was scored according to the Metavir system [13]. $\mathrm{F} \geq 2$ was regarded as significant fibrosis, $\mathrm{F} \geq 3$ as advanced fibrosis and F4 as cirrhosis.

\section{Transient elastography (Fibroscan)}

United Fibroscan devices (FS402, Echosens, France) were used for measuring liver stiffness according to the manufacturer's protocol [14]. The transient elastography (TE) results were expressed in kilopascal $(\mathrm{kPa})$ and the final measuring result was the median value of 10 successful acquisitions.

\section{Serum index calculation}

Because of simple formulas and easily available parameters, aspartate transaminase (AST)-to-platelet ratio index (APRI) and the fibrosis-4 (FIB-4) were frequently used $[15,16]$. Formulas were as follows:

$$
\begin{aligned}
& \mathrm{APRI}=\frac{(\mathrm{AST}(\mathrm{IU} / \mathrm{L}) / \mathrm{ULN}) \times 100}{\text { Platelet count }\left(10^{9} / \mathrm{L}\right)} \\
& \mathrm{FIB}-4=\frac{\text { age }(\text { years }) \times \mathrm{AST}(\mathrm{IU} / \mathrm{L})}{\text { Platelet count }\left(10^{9} / \mathrm{L}\right) \times \mathrm{ALT}(\mathrm{IU} / \mathrm{L})^{\wedge} 1 / 2}
\end{aligned}
$$

\section{Measurement of CK18 in patients}

During liver biopsy, a blood sample was obtained from each patient and processed to plasma (stored at $-80^{\circ} \mathrm{C}$ ). CK18 was quantitatively measured using the M30-Apoptisense ELISA kit (PEVIVA; Alexis, Grünwald, Germany) from the plasma. All measurements were performed in triplicate and the absorbance was determined using a microplate reader (Molecular Devices M2, Sunnyvale, CA).

\section{Sample size calculation}

The sample size was calculated according to 10 events per variable (EPV). The calculation was done based on the assumption that the possible significant variables including ALT, AST, GGT, PLT and CK18 according to previous studies about non-invasive tests for evaluation of liver disease severity and prognosis. A total of 5 variables were considered as possibly significant. Then, the estimated minimum sample size was 100.

\section{Statistical analysis}

Continuous data were presented as means with standard deviations or medians with percentiles (25th and 75th), and the comparisons between two groups were analyzed by the Mann-Whitney $U$ test. Count data were presented as percentages and the comparison was performed by the $\chi^{2}$ test. Pearson and spearman correlation analysis were used for categorical and continuous data, respectively. Forward conditional linear multivariate analysis was used to capture independent correlations for multivariate data. All combination models were established using logistic regression in the training cohort. The optimal cutoff value was determined due to the receiver operating characteristic (ROC) curve by maximizing the Youden index (sensitivity + specificity -1$)$. The validation cohort tested the model using the formula and optimal cutoff values derived from the primary cohort. The discrimination ability of models was quantified by the ROC curve and area under the curve (AUC) value. Delong test was used to compare AUC values. Decision curve analysis (DCA) was used to calculate the net benefit from the models at different threshold probabilities. A two-sided $P$ value less than 0.05 was considered statistically significant.

\section{Results}

\section{Main characteristics for CHC-T2DM patients}

In total, 252 patients between January 2012 and August 2017 were finally included: 132 (between January 2012 and September 2015) were allocated for training and 120 (between October 2015 and August 2017) for internal validation. As summarized in Table 1, no differences were observed in CK18 and other clinical-pathological characteristics between the training and validation cohorts. The overall rate of significant fibrosis, advanced fibrosis and cirrhosis was $77.0 \%$ (194 of 252), 58.7\% (148 of 252) and $38.5 \%$ (97 of 252).

\section{Correlation between CK18 and Fibrosis score in the entire cohort}

As is shown in Fig. 2, the level of CK18 was found significantly positively associated with fibrosis scores (Spearman correlation analysis, $r=0.452, P<0.001$ ). The CK18 of patients in F0, F1, F2, F3 and F4 fibrosis group was $76.6 \pm 10.9(\mathrm{ng} / \mathrm{L}), \quad 87.1 \pm 9.7(\mathrm{ng} / \mathrm{L}), 103.0 \pm 13.3$ $(\mathrm{ng} / \mathrm{L}), \quad 101.8 \pm 18.4(\mathrm{ng} / \mathrm{L})$ and $111.3 \pm 32.2(\mathrm{ng} / \mathrm{L})$, respectively. TE had the ability of reflecting the actual 
Table 1 Main characteristics

\begin{tabular}{|c|c|c|c|}
\hline Parameter & Training $(n=132)$ & $\begin{array}{l}\text { Validation } \\
(n=120)\end{array}$ & $P$ value \\
\hline Sex & & & .614 \\
\hline No. of men & $72(54.5)$ & $61(50.8)$ & \\
\hline No. of women & $60(45.5)$ & $59(49.2)$ & \\
\hline Age & & & .703 \\
\hline$<60$ (years) & 76 (57.6) & $72(60.0)$ & \\
\hline$\geq 60$ (years) & $56(42.4)$ & $48(40.0)$ & \\
\hline $\mathrm{BMl}$ & $23.86 \pm 3.06$ & $23.67 \pm 2.88$ & .618 \\
\hline \multicolumn{4}{|l|}{$\begin{array}{l}\text { Laboratory } \\
\text { findings }^{\mathrm{a}}\end{array}$} \\
\hline WBC $\left(10^{9} / L\right)$ & $5.45 \pm 2.15$ & $5.20 \pm 2.04$ & .354 \\
\hline $\mathrm{FBG}(\mathrm{mmol} / \mathrm{L})$ & $8.6 \pm 4.2$ & $9.3 \pm 5.4$ & .250 \\
\hline FINS (mIU/L) & $14.86 \pm 10.37$ & $16.33 \pm 11.49$ & .287 \\
\hline AST (IU/mL) & $57.59 \pm 68.80$ & $69.88 \pm 121.65$ & .319 \\
\hline $\mathrm{ALT}(\mathrm{IU} / \mathrm{mL})$ & $86.45 \pm 145.88$ & $87.22 \pm 169.00$ & .969 \\
\hline GGT (IU/mL) & $92.16 \pm 118.81$ & $77.40 \pm 106.36$ & .302 \\
\hline $\mathrm{ALP}(\mathrm{IU} / \mathrm{mL})$ & $98.39 \pm 60.50$ & $87.78 \pm 67.94$ & .191 \\
\hline $\begin{array}{l}\text { Platelet count } \\
\left(10^{9} / \mathrm{L}\right)\end{array}$ & $151.96 \pm 59.70$ & $148.97 \pm 68.49$ & .711 \\
\hline $\begin{array}{l}\text { Cholesterol } \\
(\mathrm{mmol} / \mathrm{L})\end{array}$ & $3.96 \pm 1.00$ & $4.04 \pm 0.92$ & .527 \\
\hline TG (mmol/L) & $1.80 \pm 1.21$ & $1.60 \pm 1.05$ & .106 \\
\hline INR & $1.17 \pm 0.18$ & $1.17 \pm 0.15$ & .774 \\
\hline APRI & $1.17 \pm 1.58$ & $1.52 \pm 3.31$ & .275 \\
\hline FIB-4 & $2.16 \pm 1.67$ & $2.69 \pm 3.42$ & .117 \\
\hline $\begin{array}{l}\text { Histologic activity } \\
\text { grade }\end{array}$ & & & .271 \\
\hline $\mathrm{A} 0$ & $27(20.5)$ & $23(19.2)$ & .875 \\
\hline $\mathrm{A} 1$ & $49(37.1)$ & $57(47.5)$ & .099 \\
\hline $\mathrm{A} 2$ & $40(30.3)$ & $32(26.7)$ & .577 \\
\hline $\mathrm{A} 3$ & $16(12.1)$ & $8(6.7)$ & .197 \\
\hline $\begin{array}{l}\text { Histologic fibrosis } \\
\text { stage }\end{array}$ & & & .787 \\
\hline F0 & $11(8.3)$ & $10(8.3)$ & $>.99$ \\
\hline F1 & $16(12.1)$ & $21(17.5)$ & .285 \\
\hline $\mathrm{F} 2$ & 25 (18.9) & $21(17.5)$ & .871 \\
\hline F3 & $29(22.0)$ & $22(18.3)$ & .531 \\
\hline F4 & $51(38.6)$ & $46(38.3)$ & .961 \\
\hline TE (kPa) & $11.13 \pm 5.21$ & $11.05 \pm 5.87$ & .914 \\
\hline CK18 (ng/L) & $105.41 \pm 28.33$ & $103.24 \pm 26.67$ & .533 \\
\hline
\end{tabular}

Except where indicated, data are numbers of patients, with percentages in parentheses

ALT, alanine transferase; ALP, alkaline phosphatase; APRI, aspartate transaminase-to-platelet ratio; AST, aspartate transaminase; BMI, body mass index; CK18, cytokeratin 18; FBG, fasting blood glucose; FINS, fasting insulin; FIB-4, fibrosis-4 index; GGT, $\gamma$-glutamyl transferase; INR, international normalized ratio; $\mathrm{TE}$, transient elastography; $\mathrm{TG}$, triglyceride; $\mathrm{WBC}$, white blood cell

${ }^{\text {a }}$ Data are presented as means \pm standard deviations

progression of liver fibrosis (Pearson correlation analysis, $r=0.855, P<0.001)$. The significant positive correlation between CK18 and TE (Pearson correlation analysis, $r=0.325, P<0.001)$ also provided additional evidence of
Table 2 Clinical characteristics of the training cohort related to fibrosis stage

\begin{tabular}{|c|c|c|c|c|}
\hline \multirow[t]{2}{*}{ Variables } & \multicolumn{2}{|c|}{$\begin{array}{l}\text { Spearman } \\
\text { correlation analysis }\end{array}$} & \multicolumn{2}{|c|}{ Multivariable analysis } \\
\hline & $r^{2}$ value & $P$ value & $b$ coefficient & $P$ value \\
\hline Age (years) & 0.018 & .124 & NA & NA \\
\hline Sex (male, female) & 0.023 & .085 & NA & NA \\
\hline BMI & 0.001 & .743 & NA & NA \\
\hline Child-Pugh $(A, B+C)$ & 0.003 & .142 & NA & NA \\
\hline $\operatorname{ALT}(\mathrm{U} / \mathrm{L})$ & 0.001 & .761 & NA & NA \\
\hline AST (U/L) & 0.021 & .146 & NA & NA \\
\hline ALP (U/L) & 0.018 & .124 & NA & NA \\
\hline GGT (U/L) & 0.024 & .076 & NA & NA \\
\hline TB $(\mu \mathrm{mol} / \mathrm{L})$ & 0.019 & .117 & NA & NA \\
\hline CB $(\mu \mathrm{mol} / \mathrm{L})$ & 0.031 & .102 & NA & NA \\
\hline ALB (g/L) & 0.085 & .001 & -0.030 & .008 \\
\hline GLOB (g/L) & 0.013 & .064 & NA & NA \\
\hline CRP (mg/L) & 0.009 & .088 & NA & NA \\
\hline WBC $\left(10^{9} / \mathrm{L}\right)$ & 0.038 & .024 & NA & .916 \\
\hline $\operatorname{PLT}\left(10^{9} / \mathrm{L}\right)$ & 0.189 & $<.001$ & NA & .279 \\
\hline $\mathrm{PT}(\mathrm{s})$ & 0.092 & $<.001$ & NA & .304 \\
\hline INR & 0.121 & $<.001$ & NA & .198 \\
\hline Cholesterol (mmol/L) & 0.000 & .914 & NA & NA \\
\hline TG $(\mathrm{mmol} / \mathrm{L})$ & 0.026 & .065 & NA & NA \\
\hline HDL-C & 0.005 & .402 & NA & NA \\
\hline LDL-C & 0.002 & .688 & NA & NA \\
\hline FBG (mmol/L) & 0.016 & .153 & NA & NA \\
\hline FINS (mIU/L) & 0.015 & .048 & NA & .234 \\
\hline IR & 0.012 & .026 & NA & .131 \\
\hline $\mathrm{TE}(\mathrm{kPa})$ & 0.736 & $<.001$ & 0.189 & $<.001$ \\
\hline CK18 (ng/L) & 0.211 & $<.001$ & 0.009 & $<.001$ \\
\hline
\end{tabular}

$b$ coefficients are from multivariable linear regression

$A L B$, albumin; ALP, alkaline phosphatase; ALT, alanine aminotransferase; AST, aspartate aminotransferase; BMI, body mass index; CK18, cytokeratin 18 ; CRP, C reactive protein; FBG, fasting blood glucose; FINS, fasting insulin; GGT, $\gamma$-glutamyl transferase; GLOB, globulin; HDL-C, high density lipoprotein cholesterol; INR, international normalized ratio; IR, insulin resistance; LDL-C, low density lipoprotein cholesterol; $\mathrm{TE}$, transient elastography; $\mathrm{TG}$, triglyceride; $\mathrm{PLT}$ blood platelet; PT, prothrombin time; TB, serum total bilirubin; WBC, white blood cell

the relation between CK18 and histologic fibrosis score. In addition, the level CK18 is positively associated with HOMA-IR (Pearson correlation analysis, $r=0.160$, $P=0.025)$.

\section{Independent predictors for liver fibrosis}

In initial spearman correlation analysis (Table 2), ALB, WBC, PLT, PT, INR, TE and CK18 were observed significantly related to fibrosis stage $(P<0.005$ for all). Through the forward conditional linear multivariable analysis, ALB, CK18 and TE were confirmed as independent predictors for the prediction of fibrosis stage 

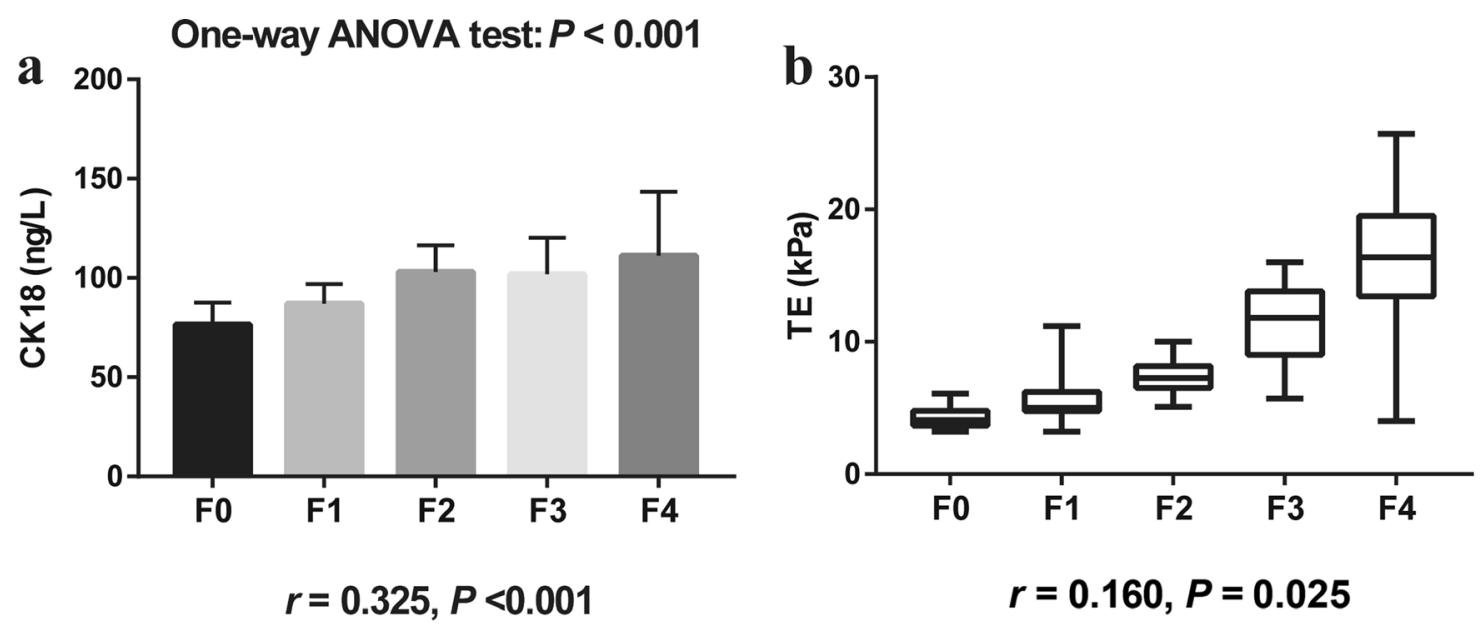

$$
r=0.160, P=0.025
$$
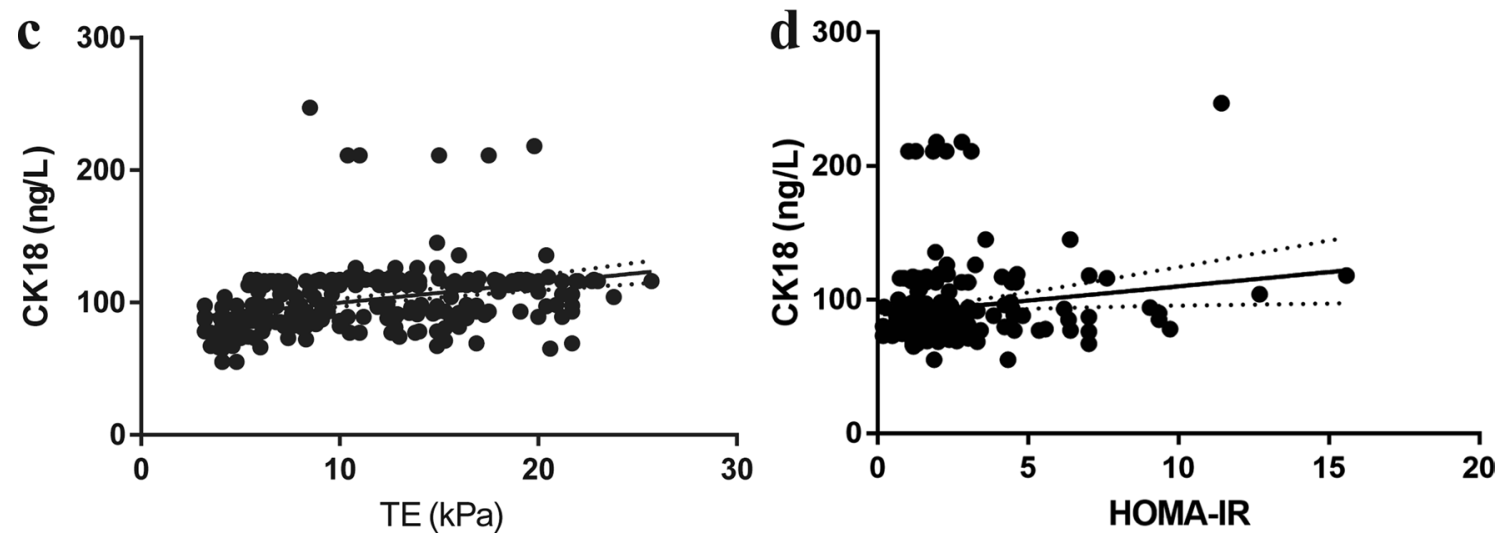

Fig. 2 Correlation analyses. a One-way ANOVA test of CK18 due to fibrosis stages; $\mathbf{b}$ Boxplots of TE due to fibrosis stages; $\mathbf{c}$ Pearson correlation analysis of CK18 and TE; d Pearson correlation analysis of CK18 and HOMA-IR. CK18, cytokeratin 18; HOMA-IR, homeostasis model assessment of insulin resistance; TE, transient elastography

Table 3 The optimal cutoff values of CK18 for the prediction of significant fibrosis, advanced fibrosis and cirrhosis

\begin{tabular}{llll}
\hline CK18 & Significant fibrosis (F0-1 vs F2-4) & Advanced fibrosis (F0-2 vs F3-4) & Cirrhosis (F0-3 vs F4) \\
\hline AUROC (95\%Cl) & $0.86(0.80,0.93)$ & $0.75(0.66,0.83)$ & $0.84(0.79,0.89)$ \\
Cutoff values (ng/L) & 90.5 & 90.5 & 104 \\
Sensitivity/specificity (\%) & $82 / 81$ & $84 / 54$ & $77 / 83$ \\
Correctly classified (\%) & 82 & 72 & 78 \\
PPV/NPV (\%) & $95 / 54$ & $74 / 68$ & $94 / 52$ \\
Positive/negative LR & $4.42 / 0.22$ & $1.81 / 0.30$ & $4.45 / 0.28$ \\
\hline
\end{tabular}

Numbers in parentheses are the $95 \%$ confidence interval

AUROC, area under the receiver operating characteristic; CK18, cytokeratin 18; LR, likelihood ratio; NPV, negative predictive value; PPV, positive predictive value

$(P=0.008,<0.001,<0.001$, respectively). As summarized in Table 3, the cutoff values of isolated CK18 for the prediction of significant fibrosis, advanced fibrosis and cirrhosis were 90.5 (ng/L), 90.5 (ng/L) and 104 (ng/L), respectively.

\section{Predictive values of CK18}

According to the cutoff values, the input values of CK18 were set as ordered categorical data $(0, \leq 90.5 \mathrm{ng} / \mathrm{L}$;
$1,>90.5 \& \leq 104 \mathrm{ng} / \mathrm{L} ; 2,>104 \mathrm{ng} / \mathrm{L})$. The formula of combination models for predicting significant fibrosis, advanced fibrosis and cirrhosis are developed from the training cohort and summarized in Table 4.

All ROC curves from the validation cohort are provided in Fig. 3. All AUC values and comparison results are summarized in Table 5. For predicting significant fibrosis, the observed AUC values of APRI + CK18 (AUC 
Table 4 The formula of established prediction methods for significant fibrosis, advanced fibrosis and cirrhosis

\begin{tabular}{llll}
\hline & Significant fibrosis (F0-1 vs F2-4) & Advanced fibrosis (F0-2 vs F3-4) & Cirrhosis (F0-3 vs F4) \\
\hline $\mathrm{TE}+\mathrm{CK} 18$ & $0.945^{*} \mathrm{TE}+1.252^{*} \mathrm{CK} 18-6.765$ & $1.003^{*} \mathrm{TE}+0.335^{*} \mathrm{CK} 18-9.157$ & $0.465^{*} \mathrm{TE}+0.576^{*} \mathrm{CK} 18-6.903$ \\
$\mathrm{APRI}+\mathrm{CK} 18$ & $0.399^{*} \mathrm{APRI}+1.858^{*} \mathrm{CK} 18-0.680$ & $-0.022^{*} \mathrm{APRI}+1.164^{*} \mathrm{CK} 18-0.987$ & $-0.006^{*} \mathrm{APRI}+1.175^{*} \mathrm{CK} 18-2.137$ \\
$\mathrm{FIB}-4+\mathrm{CK} 18$ & $0.331^{*} \mathrm{FIB}-4+1.823^{*} \mathrm{CK} 18-0.894$ & $0.227^{*} \mathrm{FIB}-4+1.133^{*} \mathrm{CK} 18-1.452$ & $0.442^{*} \mathrm{FIB}-4+1.226^{*} \mathrm{CK} 18-3.228$ \\
\hline
\end{tabular}

Numbers in parentheses are the $95 \%$ confidence interval

AUROC, area under the receiver operating characteristic; APRI, aspartate transaminase-to-platelet ratio; CK18, cytokeratin 18; FIB-4, fibrosis-4 index; TE, transient elastography

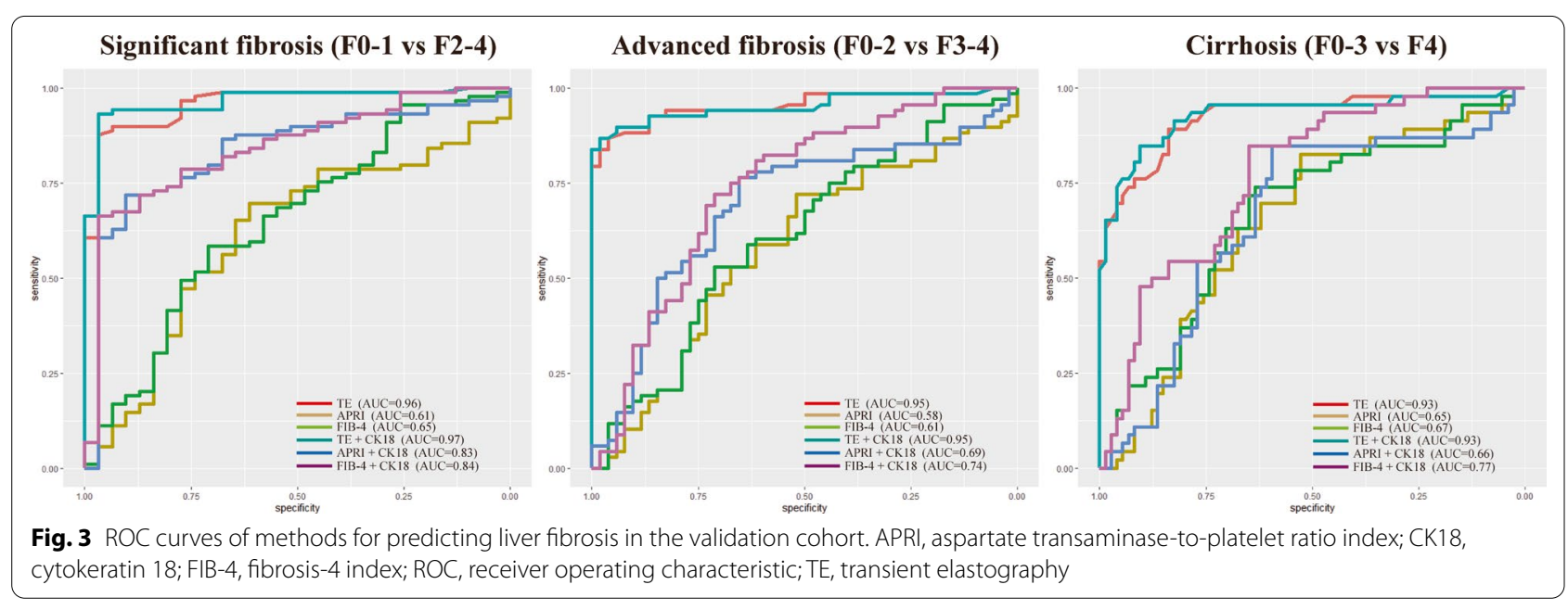

Table 5 The AUROC values of prediction methods for significant fibrosis, advanced fibrosis and cirrhosis in the validation cohort

\begin{tabular}{llll}
\hline & Significant fibrosis (F0-1 vs F2-4) & Advanced fibrosis (F0-2 vs F3-4) & Cirrhosis (F0-3 vs F4) \\
\hline TE & $0.96(0.92,0.99)$ & $0.95(0.92,0.99)$ & $0.93(0.87,0.98)$ \\
APRI & $0.61(0.50,0.73)$ & $0.58(0.47,0.68)$ & $0.65(0.55,0.75)$ \\
FIB-4 & $0.65(0.53,0.76)$ & $0.61(0.51,0.71)$ & $0.67(0.57,0.77)$ \\
TE + CK18 & $0.97(0.94,0.99)$ & $0.95(0.91,0.99)$ & $0.93(0.88,0.98)$ \\
APRI+ CK18 & $0.83(0.75,0.92)$ & $0.69(0.59,0.79)$ & $0.66(0.56,0.77)$ \\
FIB-4+ CK18 & $0.84(0.77,0.92)$ & $0.74(0.65,0.83)$ & $0.77(0.69,0.86)$ \\
Comparison of AUROC & & & .60 \\
TE and TE+CK18 & .32 & .19 & .40 \\
APRI and APRI + CK18 & $<.001$ & .02 & .89 \\
FIB-4 and FIB-4+CK18 & $<.001$ & & .03 \\
\hline
\end{tabular}

Numbers in parentheses are the $95 \%$ confidence interval

AUROC, area under the receiver operating characteristic; APRI, aspartate transaminase-to-platelet ratio; CK18, cytokeratin 18; FIB-4, fibrosis-4 index; TE, transient elastography

$0.83[95 \%$ CI $0.75,0.92])$ and FIB-4+CK18 (AUC 0.84 [95\% CI 0.77, 0.92]) were higher than those of APRI (AUC 0.61 [95\% CI 0.50, 0.73]; $P<0.001$ ) and FIB-4 (AUC 0.65 [95\% CI 0.53, 0.76]; $P<0.001$ ). For predicting advanced fibrosis and cirrhosis, the AUC values of FIB-4+CK18 (AUC 0.74 [95\% CI 0.65, 0.83], 0.77 [95\% CI $0.69,0.86]$, respectively) were significantly higher than those of FIB-4 (AUC 0.61 [95\% CI 0.51, 0.71], $P=0.02$; 0.61 [95\% CI $0.51,0.71$ ] $P=0.03$ ). No significant differences between TE + CK18 and TE were observed.

The decision curve analysis for the diagnostic methods are presented in Fig. 4. Compared with scenarios in which no prediction model would be used (i.e. treat-all or treat-none scheme), the TE or TE + CK18 provides a 


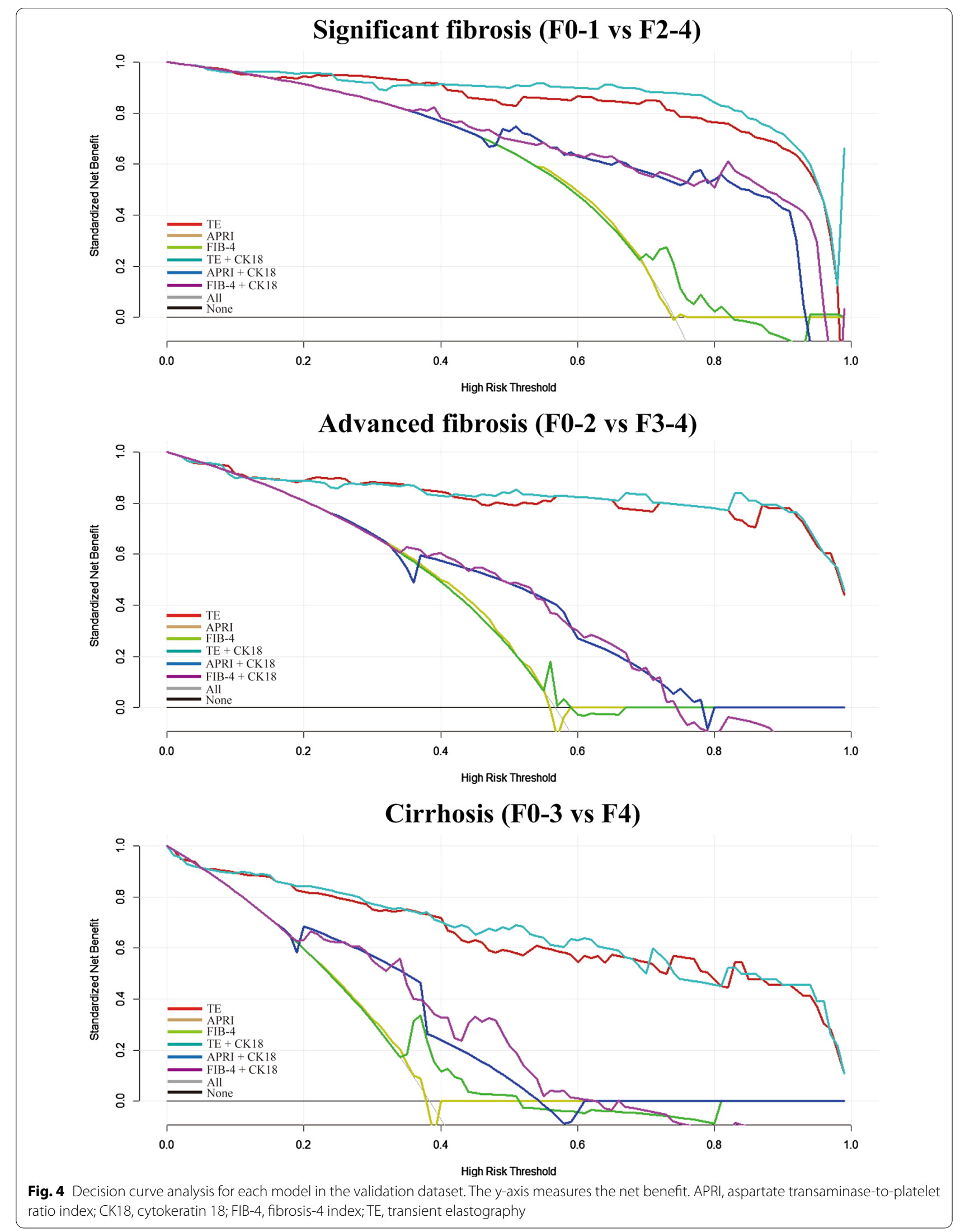


better net benefit to predict fibrosis than the other models for threshold probabilities of more than 10\%. Compared with isolated serum biomarker indices (APRI or FIB-4), APRI + CK18 or FIB- $4+$ CK18 provides more benefits for threshold probabilities of more than $40 \%$.

\section{Discussion}

In this study, we found the positive correlation between the level of CK18 and liver fibrosis stage (Spearman correlation analysis, $r=0.452, P<0.001)$ in $\mathrm{CHC}$ patients with T2DM. CK18, TE and ALB were confirmed as independent predictors for liver fibrosis. In addition, combination Models that incorporate the previously proposed methods (APRI, FIB-4 and TE) and CK were established and validated for the prediction of significant fibrosis, advanced fibrosis and cirrhosis. Promisingly, the corresponding combination models of serum biomarker indices (APRI and FIB-4) showed better discrimination ability and more clinical benefits, thereby providing important information for medical decision support.

Our results demonstrated that CK18 has the predictive value for liver fibrosis in CHC patients with T2DM. For serum biomarker indices, CK18 can contribute to diagnosing the fibrosis stage. For liver stiffness measurement, CK18 cannot significantly improve the diagnostic performance due to enough favorable performance of $\mathrm{TE}$ $[17,18]$. However, the Fibroscan device is still expensive and requires annual maintenance $(€ 34,000$ for a portable device and $€ 5000$ for its annual maintenance). In China, the machine is often accessible in the main hospitals [19]. Therefore, CK18 as an inexpensive alternative biomarker, can be combined with serum indices as inexpensive alternative methods for identifying patients with $\mathrm{CHC}$ and T2DM who need treatment.

CK18 is the predominant intermediate filament protein in the liver and contribute to substrates of caspases during hepatocyte apoptosis [20]. Levels of CK18 have been shown to be elevated in hepatocellular carcinoma, viral hepatitis, alcoholic hepatitis, NAFLD and cholestatic liver disease [21]. George et al. [22] reported that serum apoptotic caspase activity is associated with the severity of liver histologic lesions in both CHC and NAFLD.

In addition, $\mathrm{HCV}$ infection leads to a defect in insulin receptor substrate (IRS)-1 association with the IR and insulin signaling defects in hepatic IRS-1 tyrosine phosphorylation and phosphatidylinositol 3-kinase (PI3-kinase) association/activation, which contribute to insulin resistance [23]. Moreover, insulin resistance accompanied with the type 2 diabetes mellitus is positively associated with hepatic steatosis, causing an increased risk of liver fibrosis [24]. In this study, although the significant correlations between IR and CK18 $(r=0.160, P=0.025)$ or liver fibrosis $(r=0.110, P=0.026)$ were observed through correlation analysis, HOMA-IR is not confirmed as an independent predictor for liver fibrosis in $\mathrm{CHC}$ with T2DM. No significant correlation was found between CK18 and histologic liver steatosis. These might indicate that the insulin resistance might partly account for (not via liver steatosis) the correlation between CK18 and liver fibrosis. A study conducted by Jazwinski et al. [25] also demonstrated the similar conclusion that $\mathrm{CK} 18$ in $\mathrm{CHC}$ is related to advanced fibrosis but not steatosis. CK18 might be a indicator not only for liver fibrosis but also for insulin resistance in $\mathrm{CHC}$ with T2DM, which requires more large sample-size studies to investigate.

Several limitations should be noted in this study. First, inherent selection biases cannot be avoided due to the retrospective nature of this study. For instance, patients with unclear or unsatisfied results of noninvasive tests would accept liver biopsy. Absence of control group is $\mathrm{HCV}$ patients without T2DM is also a major limitation. We cannot conclude that the predictive value of CK18 is only special for CHC with T2DM. Further study should add the control group for more analyses. Finally, the combination models were established and validated on the basis of data obtained from a single center. Multi-institutional studies are required for further validations.

In conclusion, CK18 is an independent predictor of liver fibrosis for $\mathrm{CHC}$ patients with T2DM. It can provide added values to noninvasive methods for diagnosing fibrosis and help clinical decision-making.

\section{Acknowledgements \\ We would like to express our great appreciation to the R Development Core Team and contributors for $\mathrm{R}$ packages used in our study.}

\section{Authors' contributions}

Conception and design: CW and LZ; Administrative support: LZ and QG;

Provision of study materials or patients: $F L$ and $F L$; Collection and assembly of data: FL, FL and CW; Data analysis and interpretation: CW and QG; Manuscript writing: all authors. All authors read and approved the final manuscript.

\section{Funding}

None.

\section{Availability of data and materials}

The datasets analyzed during the current study are available from the corresponding authors on reasonable request.

\section{Declarations}

\section{Ethics approval and consent to participate}

This retrospective study was approved by the institutional review board of the Fourth People's Hospital of Huai'an. The requirement for written informed consent was waived by the institutional review board of the Fourth People's Hospital of Huai'an due to its retrospective nature.

Consent for publication

Not applicable. 


\section{Competing interests}

On behalf of all authors, the corresponding author states that there is no conflict of interest.

\section{Author details}

${ }^{1}$ Department of Infectious Diseases, Institute of Hepatic Diseases, Renmin Hospital, Hubei University of Medicine, Shiyan, China. ${ }^{2}$ Huai'an Second People's Hospital, The Affiliated Huai'an Hospital of Xuzhou Medical University, Huai'an, China. ${ }^{3}$ Outpatient Department, The Second People's Hospital of Huai'an, The Affiliated Huai'an Hospital of Xuzhou Medical University, Huai'an, China. ${ }^{4}$ Department of Hepatology, The Fourth People's Hospital of Huai'an, Jiangsu, China.

Received: 14 July 2021 Accepted: 30 September 2021

Published online: 20 October 2021

\section{References}

1. World Health Organization. Guidelines for the care and treatment of persons diagnosed with chronic hepatitis C virus infection. 2018. https:// www.who.int/publications-detail/guide-care-treat-persons-diagnosedchronic-hepatitis-c-978-92-4-155034-5.

2. World Health Organization. Global report diabetes. 2016. https://www. who.int/health-topics/diabetes.

3. Noubiap JJ, Nansseu JR, Nyaga UF, et al. Global prevalence of diabetes in active tuberculosis: a systematic review and meta-analysis of data from 2.3 million patients with tuberculosis. Lancet Glob Health. 2019;7(4):e448-60.

4. Gill K, Ghazinian H, Manch R, Gish R. Hepatitis C virus as a systemic disease: reaching beyond the liver. Hepatol Int. 2016;10(3):415-23.

5. Svegliati-Baroni G, Ridolfi F, Di Sario A, et al. Insulin and insulin-like growth factor-1 stimulate proliferation and type I collagen accumulation by human hepatic stellate cells: differential effects on signal transduction pathways. Hepatology. 1999;29(6):1743-51.

6. Paradis V, Perlemuter G, Bonvoust F, et al. High glucose and hyperinsulinemia stimulate connective tissue growth factor expression: a potential mechanism involved in progression to fibrosis in nonalcoholic steatohepatitis. Hepatology. 2001;34(4 Pt 1):738-44.

7. Wieckowska A, Zein NN, Yerian LM, Lopez AR, McCullough AJ, Feldstein AE. In vivo assessment of liver cell apoptosis as a novel biomarker of disease severity in nonalcoholic fatty liver disease. Hepatology. 2006;44(1):27-33.

8. Wong WW, Adams LA, de Lédinghen V, Wong GL, Sookoian S. Noninvasive biomarkers in NAFLD and NASH - current progress and future promise. Nat Rev Gastroenterol Hepatol. 2018;15(8):461-78.

9. Vilar-Gomez E, Chalasani N. Non-invasive assessment of non-alcoholic fatty liver disease: clinical prediction rules and blood-based biomarkers. J Hepatol. 2018;68(2):305-15.

10. Sanyal A, Cusi K, Hartman ML, et al. Cytokeratin-18 and enhanced liver fibrosis scores in type 1 and type 2 diabetes and effects of two different insulins. J Investig Med. 2018;66(3):661-8.

11. Darweesh SK, AbdEIAziz RA, Abd-EIFatah DS, et al. Serum cytokeratin-18 and its relation to liver fibrosis and steatosis diagnosed by FibroScan and controlled attenuation parameter in nonalcoholic fatty liver disease and hepatitis C virus patients. Eur J Gastroenterol Hepatol. 2019;31(5):633-41.

12. European Association for Study of Liver, Asociacion Latinoamericana para el Estudio del Higado. EASL-ALEH Clinical Practice Guidelines: non-invasive tests for evaluation of liver disease severity and prognosis. J Hepatol. 2015;63(1):237-64.

13. Bedossa P, Poynard T. An algorithm for the grading of activity in chronic hepatitis C. The METAVIR cooperative study. Group Hepatol. 1996:24(2):289-93.

14. Sandrin L, Fourquet B, Hasquenoph JM, Yon S, Fournier C, Mal F, Christidis C, Ziol M, Poulet B, Kazemi F, Beaugrand M, Palau R. Transient elastography: a new noninvasive method for assessment of hepatic fibrosis. Ultrasound Med Biol. 2003;29(12):1705-13.

15. Wai CT, Greenson JK, Fontana RJ, Kalbfleisch JD, Marrero JA, Conjeevaram HS, Lok AS. A simple noninvasive index can predict both significant fibrosis and cirrhosis in patients with chronic hepatitis C. Hepatology. 2003;38(2):518-26.

16. Vallet-Pichard A, Mallet V, Pol S. FIB-4: a simple, inexpensive and accurate marker of fibrosis in HCV-infected patients. Hepatology. 2006;44(3):769.

17. Castera L, Forns $X$, Alberti A. Non-invasive evaluation of liver fibrosis using transient elastography. J Hepatol. 2008;48(5):835-47.

18. Corpechot C, Carrat F, Poujol-Robert A, et al. Noninvasive elastographybased assessment of liver fibrosis progression and prognosis in primary biliary cirrhosis. Hepatology. 2012;56(1):198-208.

19. Lemoine M, Shimakawa Y, Nayagam S, et al. The gamma-glutamyl transpeptidase to platelet ratio (GPR) predicts significant liver fibrosis and cirrhosis in patients with chronic HBV infection in West Africa. Gut. 2016;65(8):1369-76

20. Linder S, Havelka AM, Ueno T, Shoshan MC. Determining tumor apoptosis and necrosis in patient serum using cytokeratin 18 as a biomarker. Cancer Lett. 2004;214(1):1-9.

21. Yilmaz Y. Systematic review: caspase-cleaved fragments of cytokeratin 18-the promises and challenges of a biomarker for chronic liver disease. Aliment Pharmacol Ther. 2009;30(11-12):1103-9.

22. Papatheodoridis GV, Hadziyannis E, Tsochatzis E, et al. Serum apoptotic caspase activity in chronic hepatitis $C$ and nonalcoholic Fatty liver disease. J Clin Gastroenterol. 2010;44(4):e87-95.

23. Aytug S, Reich D, Sapiro LE, Bernstein D, Begum N. Impaired IRS-1/ $\mathrm{PI3}-$ kinase signaling in patients with HCV: a mechanism for increased prevalence of type 2 diabetes. Hepatology. 2003;38(6):1384-92.

24. Sanyal AJ. Role of insulin resistance and hepatic steatosis in the progression of fibrosis and response to treatment in hepatitis C. Liver Int. 2011;31(Suppl 1):23-8.

25. Jazwinski AB, Thompson AJ, Clark PJ, Naggie S, Tillmann HL, Patel K. Elevated serum CK18 levels in chronic hepatitis $C$ patients are associated with advanced fibrosis but not steatosis. J Viral Hepat. 2012;19(4):278-82.

\section{Publisher's Note}

Springer Nature remains neutral with regard to jurisdictional claims in published maps and institutional affiliations.

Ready to submit your research? Choose BMC and benefit from

- fast, convenient online submission

- thorough peer review by experienced researchers in your field

- rapid publication on acceptance

- support for research data, including large and complex data types

- gold Open Access which fosters wider collaboration and increased citations

- maximum visibility for your research: over 100M website views per year

At $\mathrm{BMC}$, research is always in progress.

Learn more biomedcentral.com/submissions 